\title{
Associations of Diet and Plasma BCAA Concentrations in Healthy Men and Women - Results from the KarMeN Study
}

\author{
Benedikt Merz, Manuela Rist, Achim Bub and Bernhard Watzl \\ Max Rubner-Institut, Department of Physiology and Biochemistry of Nutrition, Karlsruhe, Germany
}

\section{Abstract}

Diet is the only source of the essential branched-chain amino acids (BCAA) isoleucine, leucine and valine. High plasma concentrations of these amino acids are discussed as risk factors for the onset of several diseases such as type 2 diabetes mellitus (T2D) or cardiovascular diseases (CVD). Information about the contribution of the overall diet to plasma levels of these amino acids is controversial. Our objective was to investigate which dietary pattern is associated with plasma BCAA concentrations in a healthy population and which other additional nutrients besides isoleucine, leucine and valine, such as other amino acids, may contribute to the diseases risk.

The Karlsruhe Metabolomics and Nutrition (KarMeN) study is a cross-sectional study aiming to determine the impact of a number of factors on the human metabolome in healthy men and women aged 18 and 80 years. In our study, fasting plasma amino acid concentrations as well as current and habitual dietary intake were assessed in 298 apparently healthy individuals, 171 men (57.4\%) and 127 women $(42.6 \%)$ with a mean age of 44.5 and 51.6 years, respectively. All reported foods were summarized into 35 food groups. Dietary patterns were derived that explain as much variation as possible in plasma BCAA concentrations using reduced rank regression. The first derived current dietary pattern covering the diet of the past 24 hours, showed $19.2 \%$ of explained variance for BCAA plasma concentrations, whereas the first habitual dietary pattern, covering a period of more than 4 weeks, explained $32.5 \%$. For further analysis, we focused on the first derived habitual dietary pattern. This pattern was high in meat, sausages, sauces, eggs, and ice cream but low in nuts, cereals, mushrooms, and pulses. The age, sex, and energy intake adjusted dietary pattern score was associated with an increase in animal-based protein and at the same a decrease in plant-based protein, dietary fibre and an unfavorable fatty acid composition. Amino acids alanine, lysine and the aromatic amino acids phenylalanine, tyrosine, and tryptophan were positively associated with the dietary pattern score as well. All of these factors were reported to be associated with risk of T2D and CVD.

Our data suggest that rather than the dietary intake of BCAA, the overall dietary pattern contributing to high BCAA plasma concentrations may modulate the chronic diseases risk.

\section{Conflict of Interest}

There is no conflict of interest. 